\title{
An Identification of the unbalanced magnetic pull in gener- ator at excitation and the hydropower machine model vali- dation
}

\author{
Željan Lozina ${ }^{1, *}$, Damir Sedlar ${ }^{1, * *}$, and Ivan Tomac ${ }^{1, * * *}$ \\ ${ }^{1}$ University of Split - FESB, Ruđera Boškovića 32, HR-21000 Split, Croatia
}

\begin{abstract}
A mechanical vibration inverse analysis has been performed on 150MW hydro-power machine in order to identify unbalanced magnetic pull. FEM model of the machine is developed according to design data. The System Equivalent Reduction Expansion Process is involved in model validation during the power-machine experimental run. The unbalanced magnetic pull in the generator is calculated from the verified model and monitored data.
\end{abstract}

\section{Introduction}

The hydropower machinery is exposed to vibration during operation as result of many excitation sources. The reliable simulation of the dynamical behavior is strongly dependent on the mathematical models of hydropower machine and corresponding excitation. The topics related to these subjects are extensively researched until nowadays, [1-5], and still keep attention of the researchers.

The unbalanced magnetic pull (UMP) in power machinery are subject of the recent research. In [6] has been developed a general theory for the UMP and the analytical expression is provided for the UMP that consists of harmonic components. Also, to verify the theoretical results, in [7] the radial stability of flexible shaft in two poles induction machines is investigated. More recently, in [8] the analytical expression of the UMP in a three-phase electrical machine with any numbers of pole-pairs has been derived. The approach advances the idea of modulating the fundamental magneto-motoric force wave by air-gap permanence expressed as a Fourier series.

In [9] and [10] an analytical model of hydro power units have developed in order to determine the UMP and thus obtained results have been compared with FEM simulation. The saturation effects of the ferromagnetic materials have been taken into consideration, too [9]. The influence of the UMP on the rotor using linear and nonlinear model of the generator has been researched in [10] where an improved reduction technique was used to reduce the degrees of freedom of FE model to make a comparison with measurement.

An experimental investigation of a cage induction motor with a statically eccentric rotor using piezoelectric transducers is described in [11]. The load cell incorporated into the rotor

\footnotetext{
*e-mail: lozina@fesb.hr

**e-mail: dsedlar@fesb.hr

***e-mail: itomac@fesb.hr
} 


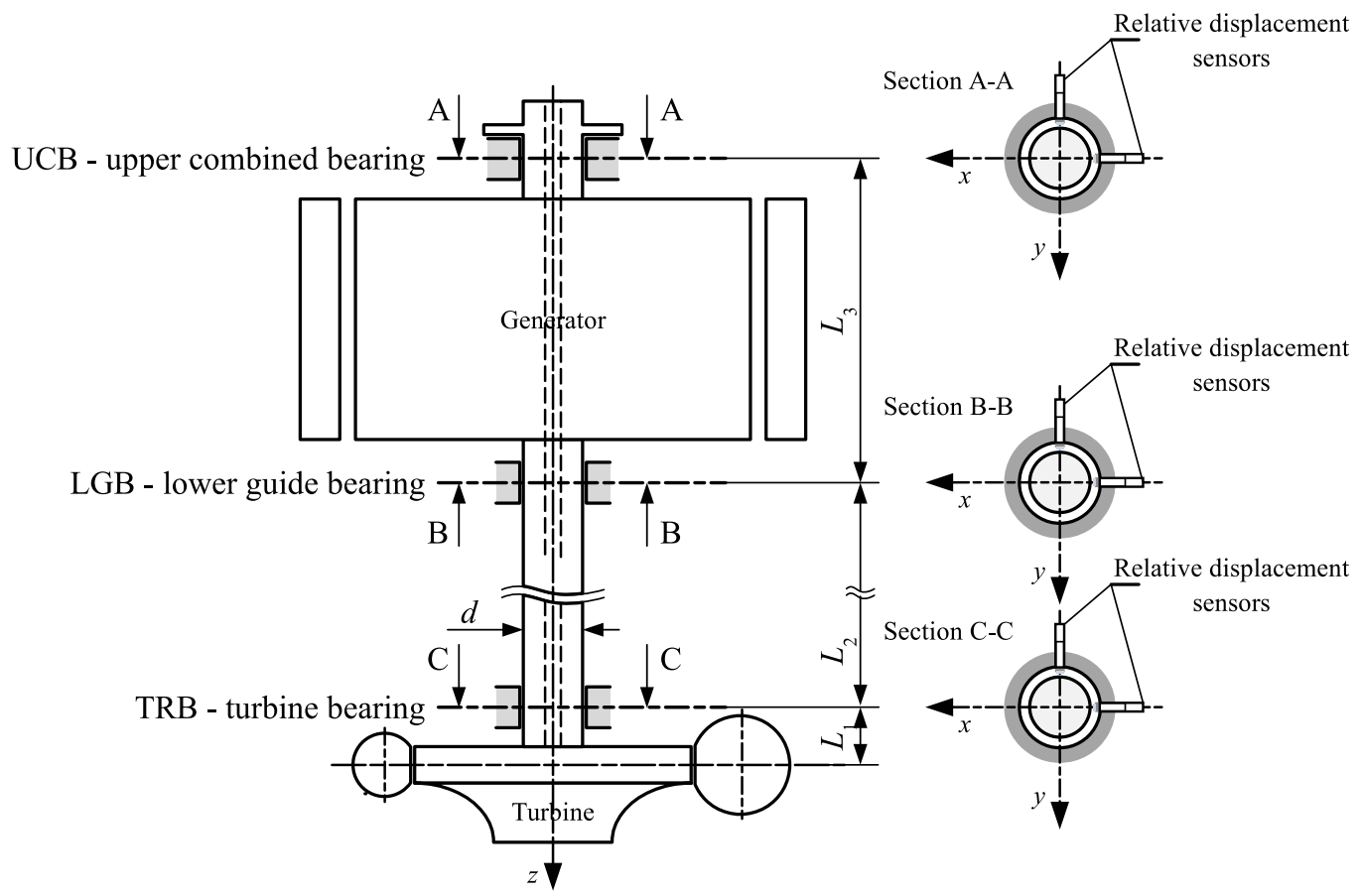

Figure 1: The hydraulic-power machine general layout.

support pedestals was used during measurement of UMP, [12]. The UMP and torque in a wound rotor induction machine when the non centered rotor is calculated.

In this paper, the UMP in the generator of a hydropower machine is identified. The identification is based on the monitored data and FEM model of the hydropower machine. The system equivalent reduction and expansion process (SEREP) is involved in order to match FEM model and experimentally retrieved partial model. It is proved that the FEM model reliably simulates the dynamic interaction between turbine and generator for the considered operational state.

\subsection{Hydro-power machine general description}

The 150 MW hydro-power machine with the vertical shaft hanged at the top is considered, Fig. 1. The shaft overall length is $13.892 \mathrm{~m}$ and machine total mass is $375 \times 10^{3} \mathrm{~kg}$. The average working had is $250 \mathrm{~m}$ and flow rate at maximum power is $60 \mathrm{~m}^{3} \mathrm{~s}^{-1}$. The rotational speed is $333 \mathrm{~min}^{-1}$. The top combined axial-radial guide bearing and two lower radial guide bearings (the upper and lower generator bearings and the turbine bearing) are of segmented design with forced lubrication. In this case (synchronic) three phase and 18 magnetic poles generator is considered. The generator rotor has ten magnetic poles (five magnetic pole couples) and the Francis turbine runner has 15 vanes. 


\subsection{Hydro-power machine model}

The modeling of hydraulic power machinery is researched extensively, [10, 13]. The special attention is addressed to the bearings, [14-16] and unbalanced magnetic pull, [11, 12, 17].

The model can be transferred to the co-rotational coordinate system i.e. the body coordinate system that synchronously rotates with the shaft. The first order of the dynamic forces and corresponding displacements in this coordinate system appears as if it is static component.

\section{Model retrieved from measurement}

Generally, the models can be retrieved from experiments correlating known-measured inputs and outputs or using outputs only (typically from operational data). In this specific case, a limited indirect control over input was available and the focus was on rotational speed related phenomena. Therefore, the order tracked data are correlated for different machine states.

\section{Theoretical background and practical limitations}

\subsection{Typical measuring set-up and machine monitoring}

The typical machine monitoring system relays on sensors collecting the accelerations of the bearing housing and the shaft displacements relative to bearing in two perpendicular directions at each bearing, see Fig. 1. The switch (at least one per turn) is used as tachometer. The sensors of axial vibrations in the axial bearing are common too.

Regarding the indication of forces, relative shaft displacements are more reliable then absolute bearing housing accelerations in considered design. The acceleration of the bearing housing is a function of the force but also it is a function of the supporting structure stiffness, therefore, low acceleration of the housing cannot exclude possible high force because the bearing force can be transferred via supporting structure to the foundation and absorbed there, compare a) and b) in Fig. 2. Although only the linear values are measured (linear acceleration, velocities and displacements) often angular quantities are used in the presentations for a number of reasons and therefore linear values are transformed into angular, i.e. forces to couples, linear to angular velocities,... The generator displacements are measured at bearings but very often they are presented as linear and angular displacement, see section 3.1.

\subsection{Model validation objectives and limits}

More specifically, we do not identify the forces directly (using force sensors) but indirectly concluding from effects that are an obvious consequence of the forces, e.g. the accelerations (and consequently the speeds and the displacements).

However, when changes are observed then processing of a difference between two known states is required. So, in order to assess the mathematical model it is necessary to subtract the displacement vectors before the magnetic excitation from the corresponding vectors after the excitation and so isolate the net excitation effect.

The common objectives of the model correlation is verification of the model and eventual a model update in order to increase model accuracy and reliability. 

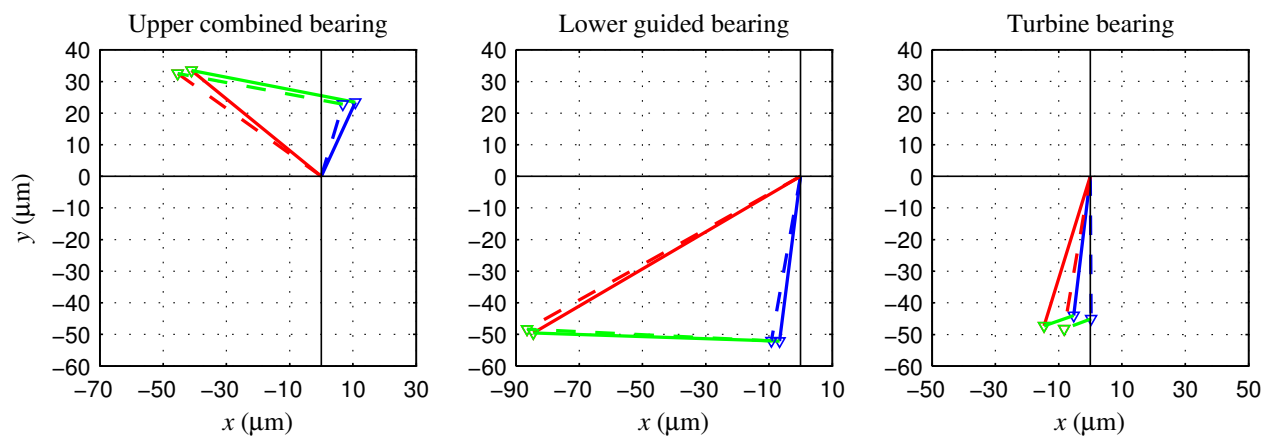

(a) Relative displacements.
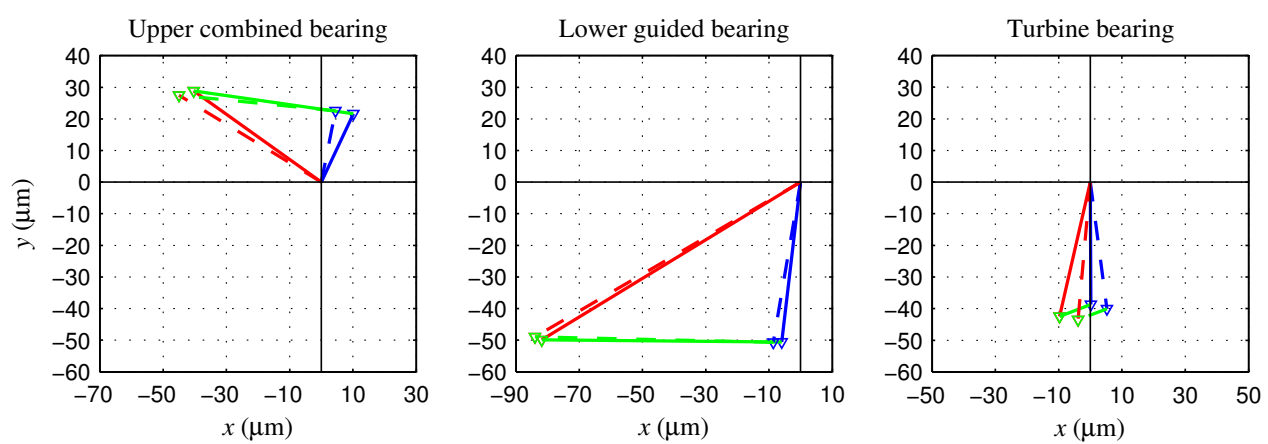

(b) Absolute displacements.

Figure 2: Influence of the generator magnetisation and mechanical run. Blue colour is the vector of mechanical run, red colour is the vector of magnetisation and green colour shows the difference between these two regimes. Solid lines include first order vector only and dashed lines include sum of first three order vectors.

\section{Experiment data processing}

The orbits clearly demonstrate the sensitivity of the machine relative shaft displacements to the power. The relation between the relative shaft displacement orders (1. order compared to 2 . orders is 10 times higher and so on) demonstrates dominant influence of co-rotational forces, see Fig. 2.

\subsection{The generator linear and angular displacement and corresponding forces}

The reduction of the updated FEM model motion equations, using SEREP [18], to 3 linear node displacements leads to the expression in matrix form, Eqn. 1.

$$
\left[\begin{array}{lll}
k_{11} & k_{12} & k_{13} \\
k_{21} & k_{22} & k_{23} \\
k_{31} & k_{32} & k_{33}
\end{array}\right]\left\{\begin{array}{c}
\mathrm{UCB}_{\mathrm{y}} \\
\mathrm{LGB}_{\mathrm{y}} \\
\mathrm{TRB}_{\mathrm{y}}
\end{array}\right\}=\left\{\begin{array}{l}
\mathrm{UCB}_{\mathrm{Fy}} \\
\mathrm{LGB}_{\mathrm{Fy}} \\
\mathrm{TRB}_{\mathrm{Fy}}
\end{array}\right\}
$$



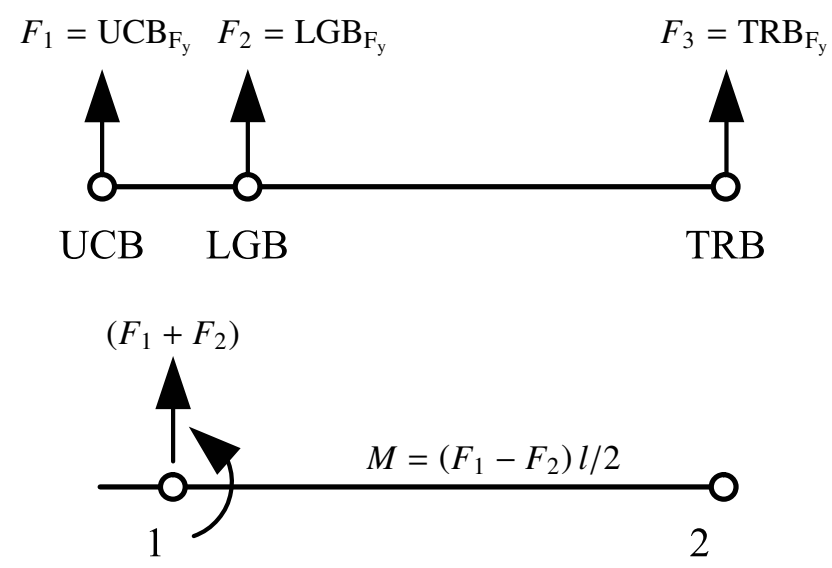

Figure 3: Magnetization and forces on the rotor and transformation to the bearing dofs.

The practical reasons related to the model of the magnetic field influence quoted as the parallel shift and angular offset, motivate us to transform the 3 linear degree of freedom to two linear and one angular degree of freedom, see Fig. 3.

After the transformation that involves a summation and a subtraction of the first two equations from the Eqn. 1 and corresponding substitutions we obtain matrix equation expressed:

$\left[\begin{array}{ccc}k_{11}+k_{22}+2 k_{21} & -\frac{L}{2}\left(k_{11}-k_{22}\right) & k_{13}+k_{23} \\ \frac{L}{2}\left(k_{11}-k_{22}\right) & \left(k_{11}+k_{22}-2 k_{12}\right) \frac{L^{2}}{4} & \frac{L}{2}\left(k_{23}-k_{13}\right) \\ k_{31}+k_{32} & \frac{L}{2}\left(k_{32}-k_{21}\right) & k_{33}\end{array}\right]\left\{\begin{array}{c}u \\ \theta \\ \mathrm{TRB}_{\mathrm{y}}\end{array}\right\}=\left\{\begin{array}{c}\mathrm{UCB}_{\mathrm{Fy}}+\mathrm{LGB}_{\mathrm{Fy}} \\ M \\ \mathrm{TRB}_{\mathrm{Fy}}\end{array}\right\}$

where $u$ and $\theta$ are linear and angular displacements, respectively. Using concrete values for the power-machine model and corresponding measurements, [19], that are post-processed according model requirements:

$$
\begin{aligned}
& u=6.2530 \times 10^{-5} \mathrm{~m}, \\
& \theta=2.801 \times 10^{-6} \mathrm{rad}, \\
& \mathrm{TRB}_{\mathrm{y}}=-7.4106 \times 10^{-6} \mathrm{~m} .
\end{aligned}
$$

the estimated forces due to magnetization can be calculated, Eqn. 2

$$
\left\{\begin{array}{c}
\mathrm{UCB}_{\mathrm{Fy}}+\mathrm{LGB}_{\mathrm{Fy}} \\
M \\
\mathrm{TRB}_{\mathrm{Fy}}
\end{array}\right\}=\left\{\begin{array}{l}
1.5187 \\
0.3478 \\
0.4341
\end{array}\right\} 10^{5}
$$

where forces are in $\mathrm{N}$ and moment is in $\mathrm{Nm}$.

The UMP is in balance with forces in machine bearings. The generator bearings take over the major part of the UMP, as seen in the last expression. The UMP couple is balanced with bearing reaction couple of the moment $M$. The turbine bearing force has low influence to the generator behavior due to relative low shaft flexible stiffness, as proved in the experiments.

\section{Conclusion}

In this paper a case study of UMP and the phenomena related to the forces in the hydro-power machine with the vertical shaft hanged at the top are identified. The previously verified FEM 
model of the hydro-power machine, reduced according SEREP to measured dof is used in assessment of the UMP.

\section{References}

1. Y. Wu, S. Li, S. Liu, H. Dou, Z. Qian, Vibration of Hydraulic Machinery (Springer, 2013)

2. A. Muszynska, Rotordynamics (Taylor and Francis, 2005)

3. H. Ohashi, Vibration and Oscillation of Hydraulic Machinery (Ashgate, 2009)

4. D. Childs, Turbomachinery Rotordynamics: Phenomena, Modeling, and Analysis (Wiley, 1993)

5. J.M. Vance, Rotordynamics of Turbomachinery (John Wiley and sons, 1988)

6. R. Belmans, W. Geysen, H. Jordan, A. Vandenput, Unbalanced Magnetic Pull in Three Phase Two Pole Induction Motors with Eccentric Rotor, in Int. Conf. Electrical Machines-Design and Application (London, 1982), pp. 65 - 69

7. R. Belmans, A. Vandenput, W. Geysen, Electric Power Applications, IEEE Proceedings B 134, 101 (1987)

8. D. Guo, F. Chu, D. Chen, J. Sound and Vibration 254, 297 (2002)

9. Y. Xu, Z. Li, J. Vib. Acoust. 134, 051013 (2012)

10. R. Gustavsson, Ph.D. thesis, Luleå University of Technology (2007)

11. D.G. Dorrell, A.C. Smith, Electric Power Applications, IEE Proceedings 143, 202 (1996)

12. D. Dorrell, O. Kayani, IEEE Transactions on Magnetics 50, 1 (2014)

13. M. Nässelqvist, Ph.D. thesis, Luleå University of Technology (2009)

14. M.Y. Cha, Ph.D. thesis, KTH Royal Institute of Technology (2015)

15. X.D. Lai, G.L. Liao, Y. Zhu, X. Zhang, Q.Q. Gou, W.B. Zhang, Lateral vibration of hydro turbine-generator rotor with varying stiffness of guide bearings, in IOP Conference Series: Earth and Environmental Science (2012), Vol. 15

16. G.F. Simmons, Ph.D. thesis, Luleå University of Technology (2013)

17. B. Han, S. Zheng, X. Liua, Mathematical Problems in Engineering (2013)

18. J. O'Callahan, P. Avitable, R. Riemer, System equivalent reduction expansion process, in Seventh Int. Modal Analysis Conf. (Las Vegas, Nevada, 1989)

19. O. Husnjak, G. Orešković, Tech. Rep. TI 15102014, Veski d.o.o. Zagreb (2014) 